\title{
Comparison of different absorption corrections on the model structure of tetrakis( $\mu_{2}$-acetato)-diaqua-di-copper(II)
}

\author{
Julia Koziskova ${ }^{a}$, Friedemann Hahn ${ }^{b}$, \\ Jens Richter ${ }^{b}$, Jozef Kožíšek ${ }^{a}$ \\ ${ }^{a}$ Faculty of Chemical and Food Technology, Slovak Technical University, \\ Radlinského 9, SK-812 37, Bratislava, Slovakia \\ ${ }^{b}$ STOE E Cie GmbH, Hilpertstrasse 10, D-64295, Darmstadt, Germany \\ julia.koziskova@stuba.sk
}

\begin{abstract}
Two different absorption correction methods were compared in order to find out which method is preferable to improve solving and refining a crystal structure. Experiments were performed on the crystal of a tetrakis( $\mu_{2}$-acetato)-diaqua-di-copper(II) complex. The first correction method used was the numerical absorption correction with the aid of a crystal-shape model, and the other was the semi-empirical one, applying scaling routines to the intensity data.
\end{abstract}

Keywords: Absorption correction; crystal structure; accuracy

\section{Introduction}

After a measurement on an X-ray single-crystal diffractometer and after deriving net intensities for the collected reflections (being corrected for Lorentzpolarization factor etc.), an absorption correction is important for obtaining accurate structure factors, if absorption of x-rays by the crystal plays a significant role. There are, in principle, three different treatments of this correction, being well described in WinGX software (Farrugia, 2012):

a) Exact numerical corrections - analytical, Gaussian quadrature, spherical and cylindrical

b) Semi-empirical corrections - psi-scans, CAMELJOCKEY and multiscan

c) Refined corrections - DIFABS, XABS2, SHELXA

In University Science Park of STU Bratislava we have obtained a new diffractometer Stoe STADIVARI. Using this diffractometer it is possible to perform the absorption correction in two ways: the user friendly semi-empirical correction, implemented in a program called LANA, and the time consuming numerical absorption correction based on a detailed shape description of the crystal. The purpose of this article is to compare these two different absorption correction methods.

Within LANA, an absorption correction can be performed on an intensity dataset by a combination of frame scaling with direction-dependent reflection scaling.

Both scaling methods need a sufficient number of groups of symmetry-related, corrected reflections in the dataset (big redundancy), assuming that finally the intensities of all members of a group should be identical, if there were no statistical variations and systematic errors. The latter may be caused by absorption of x-rays by an anisotropically shaped crystal (amongst other possible reasons).

LANA generates scaling factors by least-squares routines, in order to minimize intensity differences within the groups of symmetry-related reflections. Subsequently the scaling factors are applied to the input reflection data, and a new intensity dataset is written into an output file.

In more detail, for each of the reflections being recorded, the influence of the absorption can be split into an incident x-ray beam component and the reflected beam one with respect to the crystal. With the aid of frame scaling, the entirety of all incident-beam components of a measurement can be taken into account. For each of the frames an individual scaling factor is generated, meaning that finally all reflection intensities originating from a specific frame get a common frame-scaling factor. For each subset of the measurement, all frame-scaling factors are coupled each other by a polynomial in order to increase the robustness of the method.

The influence of the reflected-beam components is used for direction-dependent scaling which is implemented similarly to the Blessing's method (Blessing, 1995) using spherical harmonic functions. (The main difference is that in LANA such functions are usually employed for the reflectedbeam components only, but not for the incidentbeam ones.) A set of direction-dependent scaling factors is computed, which refer to the directions of the reflected beams with respect to a coordinate system coupled with the crystal. 
The numerical absorption correction needs information about the faces of the crystal. Consequently the path of the primary and diffracted beam through the crystal is calculated. The final intensity of the diffracted beam is corrected using a linear absorption coefficient and the beam path length through the crystal.

\section{Experimental}

Experiments were realized on a Stoe STADIVARI diffractometer with a Dectris Pilatus $300 \mathrm{~K}$ detector and with an Incoatec $\mathrm{I} \mu \mathrm{S} \mathrm{Ag}$ microfocus source $(\mathrm{Ag}-\mathrm{K} \alpha, \lambda=0.56083 \AA$ ) at $100 \mathrm{~K}$ using a nitrogen gas open-flow cooler Cobra from Oxford Cryosystems.

Data reduction and cell refinement were processed using X-Area (Stoe, 2016).

Two different absorption corrections (being implemented in X-Area) were employed: numerical absorption correction and scaling procedures within LANA. For the first method a crystal-shape model with 15 faces was used, which was derived from examining the crystal with the aid of a magnifying video camera and with X-View software (Stoe, 2013). In LANA a combination of frame scaling (by cubic polynomials) and direction-dependent scaling for the intensities of the reflected beams (with maximum band orders $1_{\text {even, } \max }=6$ and $l_{\text {odd, } \max }=3$ for the spherical harmonic functions) was applied.

Crystal structure was solved by charge-flipping algorithm Superflip (Palatinus and Chapuis, 2007) using OLEX2 (Dolomanov et al., 2009) and was subsequently refined using SHELXL (Sheldrick, 2015).

The positions of all hydrogen atoms were geometrically optimized and constrained to ride on their parent atoms, with $d(\mathrm{C}-\mathrm{H})=0.98 \AA$ and $d(\mathrm{O}-\mathrm{H})=0.87 \AA$ with $\mathrm{U}_{\text {iso }}(\mathrm{H})=1.5 \times \mathrm{U}_{\mathrm{eq}}(\mathrm{C})$ and $\mathrm{U}_{\text {iso }}(\mathrm{H})=1.5 \times \mathrm{U}_{\mathrm{eq}}(\mathrm{O})$, respectively. All non-hydrogen atoms were refined with anisotropic thermal parameters. Figures and tables were generated using DIAMOND (Bergerhoff et al., 1996), OLEX2 and WinGX.

\section{Results and discussion}

The crystal structure consists of a dimer (Fig. 1) where two copper central atoms are bonded together by four acetate groups. Each of the central atoms is coordinated by four oxygen atoms in an equatorial plane. A water molecule is coordinated in an apical position (Tabs. 1-2).

Cu-Cu interaction of 2.6151(3) Å completes the coordination polyhedron to hexagonal. There are strong hydrogen bonds between the water molecule and the acetate groups of the adjacent molecules (Tab. 3.).

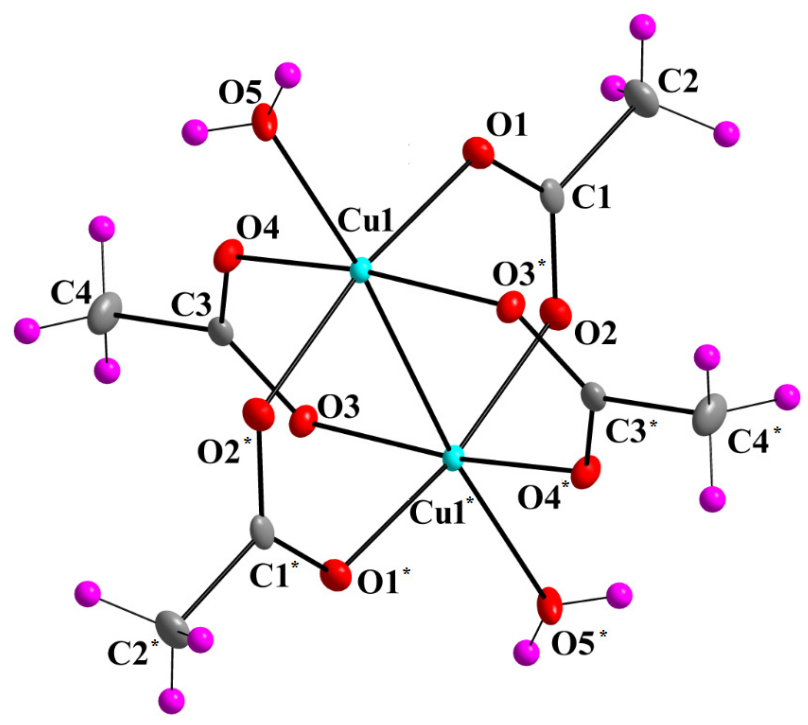

Fig. 1. The molecular structure of the studied tetrakis $\left(\mu_{2}\right.$-acetato)-diaqua-di-copper(II) complex

[symmetry code (*) 3/2-X, 1/2-Y, 1-Z].

The displacement ellipsoid plot is at the $50 \%$ probability level.

(C - grey, H - fuchsia, O - red, Cu - cyan).

In order to investigate the quality of the two absorption-correction methods applied, we compared the values of selected bonds and bond angles derived by refining the structure models obtained by employing the corresponding corrected intensity datasets. All these values showed minimal differences (Tabs. 1-3) indicating the equivalence of both methods concerning the structure of the copper complex.

There were published 14 crystal structures of tetrakis( $\mu_{2}$-acetato)-diaqua-di-copper(II) based on single crystal data. The lowest value of $\mathrm{R}$ factor was $1.50 \%$ from charge density studies with a resolution of $0.386 \AA$ (Bertolotti et al., 2012; CCDC refcode CUAQAC28)

There are six comparable routine crystal structures in CSD with $\mathrm{R}$ values equal or below $3.00 \%$ : (Golzar et al., 2007; CCDC refcode CUAQAC27) with R-factor of $2.55 \%$, (Fronczek et al., 2003; CCDC refcode CUAQAC23) with R-factor of $2.40 \%$, (Nieger et al., 2001; CCDC refcode CUAQAC05) with R-factor of $1.93 \%$, (Mahmoudkhani et al., 1998; CCDC refcode CUAQAC01) with R-factor of $1.95 \%$ and (Shamuratov et al., 1994; CCDC refcode CUAQAC04) with R-factor of $3.00 \%$.

The routine crystal structure with the lowest $\mathrm{R}$ value of $1.80 \%$ was published by Vaughan et al. (Vaughan et al., 2004; CCDC refcode CUAQAC24).

Our data obtained on the Stoe STADIVARI diffractometer show an agreement with $\mathrm{R}$ factor for the numerical method of $1.61 \%$ and for the semiempirical one $1.47 \%$ with a resolution of $0.80 \AA$ (Tabs. 4-5). 
Tab. 1. Comparison of selected bond lengths (A) for numerical (A) and semi-empirical (B) methods.

\begin{tabular}{|c|c|c|c|c|c|}
\hline & $\mathbf{A}$ & B & & $\mathbf{A}$ & B \\
\hline Cu1-Cu1* & $2.6151(3)$ & $2.6151(3)$ & O3-C3 & $1.2671(19)$ & $1.2672(18)$ \\
\hline $\mathrm{Cu} 1-\mathrm{O3}^{*}$ & $1.9875(11)$ & $1.9867(11)$ & $\mathrm{O} 2-\mathrm{C} 1$ & $1.264(2)$ & $1.264(2)$ \\
\hline $\mathrm{Cu} 1-\mathrm{O}^{*}$ & $1.9586(12)$ & $1.9591(11)$ & O4-C3 & $1.2702(19)$ & $1.2703(18)$ \\
\hline Cu1-O4 & $1.9919(11)$ & $1.9922(11)$ & O1-C1 & $1.2680(19)$ & $1.2679(18)$ \\
\hline Cu1-O5 & $2.1463(11)$ & $2.1459(11)$ & C3-C4 & $1.502(2)$ & $1.502(2)$ \\
\hline $\mathrm{Cu} 1-\mathrm{O} 1$ & $1.9450(11)$ & $1.9451(11)$ & C1-C2 & $1.506(2)$ & $1.506(2)$ \\
\hline
\end{tabular}

Symmetry code used: ${ }^{*} 3 / 2$ - X, 1/2 - Y, 1 - Z.

Tab. 2. Comparison of selected bond angles $\left(^{\circ}\right)$ for numerical (A) and semi-empirical (B) methods.

\begin{tabular}{lrrrrr}
\hline & \multicolumn{1}{c}{ A } & \multicolumn{1}{c}{ B } & \multicolumn{1}{c}{ A } & \multicolumn{1}{c}{ B } \\
\hline O2* Cu1 O3 $^{*}$ & $91.10(5)$ & $91.12(4)$ & O5 Cu1 O4 & $92.70(4)$ & $92.70(4)$ \\
O4 Cu1 O3 $^{*}$ & $169.01(4)$ & $169.01(4)$ & O1 Cu1 O3* & $87.17(5)$ & $87.17(4)$ \\
O4 Cu1 O2* $^{*}$ & $89.51(5)$ & $89.49(5)$ & O1 Cu1 O2 & $169.19(5)$ & $169.20(5)$ \\
O5 Cu1 O3 $^{*}$ & $98.21(4)$ & $98.21(4)$ & O1 Cu1 O4 & $90.17(5)$ & $90.18(4)$ \\
O5 Cu1 O2 $^{*}$ & $93.48(5)$ & $93.47(4)$ & O1 Cu1 O5 & $97.33(5)$ & $97.33(4)$ \\
\hline
\end{tabular}

Symmetry code used: *3/2 - X, 1/2 - Y, 1 - Z.

Tab. 3. Hydrogen bonds $\left(\AA^{\text {and }}{ }^{\circ}\right)$ for numerical (A) and semi-empirical (B) methods.

\begin{tabular}{|c|c|c|c|c|c|c|c|c|}
\hline & $\mathbf{A}$ & & & & B & & & \\
\hline $\mathbf{D}-\mathbf{H} \ldots \mathbf{A}$ & $\mathrm{d}(\mathrm{D}-\mathrm{H})$ & $\mathrm{d}(\mathrm{H} \ldots \mathrm{A})$ & $\mathrm{d}(\mathrm{D} \ldots \mathrm{A})$ & $<(\mathrm{DHA})$ & $\mathrm{d}(\mathrm{D}-\mathrm{H})$ & $\mathrm{d}(\mathrm{H} \ldots \mathrm{A})$ & $\mathrm{d}(\mathrm{D} \ldots \mathrm{A})$ & $<$ (DHA) \\
\hline O5-H5a $\cdots 4^{*}$ & $0.868(3)$ & $1.921(3)$ & $2.788(2)$ & $178(2)$ & $0.868(3)$ & $1.921(3)$ & $2.788(2)$ & $178(2)$ \\
\hline O5-H5b…03 ${ }^{* *}$ & $0.868(3)$ & $2.034(6)$ & $2.888(2)$ & $168(2)$ & $0.868(3)$ & $2.034(5)$ & $2.889(2)$ & $169(2)$ \\
\hline
\end{tabular}

Symmetry code used: * $1-\mathrm{X},+\mathrm{Y}, 1 / 2-\mathrm{Z} ;{ }^{* *}-1 / 2+\mathrm{X}, 1 / 2+\mathrm{Y},+\mathrm{Z}$.

Tab. 4. Experimental details.

\begin{tabular}{|c|c|}
\hline Empirical formula & $\mathrm{C}_{2} \mathrm{H}_{4} \mathrm{O}_{2.5} \mathrm{Cu}_{0.5}$ \\
\hline Formula weight & 399.30 \\
\hline Temperature (K) & 100.0 \\
\hline Crystal system & monoclinic \\
\hline Space group & $\mathrm{C} 2 / \mathrm{c}$ \\
\hline $\mathrm{a}, \mathrm{b}, \mathrm{c}(\AA)$ & $13.0989(2), 8.5127(1), 14.0602(2)$ \\
\hline$\alpha, \beta, \gamma\left(^{\circ}\right)$ & $90,119.302(1), 90$ \\
\hline Volume $\left(\AA^{3}\right)$ & $1367.21(4)$ \\
\hline $\mathrm{Z}$ & 4 \\
\hline Diffractometer & Stoe STADIVARI \\
\hline Lin. abs. coefficient $\left(\mathrm{mm}^{-1}\right)$ & 1.659 \\
\hline $\mathrm{T}_{\min }, \mathrm{T}_{\max }$ & $0.7429,0.8655$ \\
\hline$\rho_{\text {calc }}\left(\mathrm{g} / \mathrm{cm}^{3}\right)$ & 1.9397 \\
\hline $\mathrm{F}(000)$ & 810.9 \\
\hline Crystal size $\left(\mathrm{mm}^{3}\right)$ & $0.210 \times 0.145 \times 0.135$ \\
\hline Radiation type & $\operatorname{Ag} \operatorname{K} \alpha(\lambda=0.56083 \AA)$ \\
\hline $2 \Theta$ range for data collection $\left(^{\circ}\right)$ & 4.68 to 41.04 \\
\hline Index ranges & $-22 \leq \mathrm{h} \leq 22,-14 \leq \mathrm{k} \leq 14,-23 \leq 1 \leq 23$ \\
\hline Reflections collected & 20344 \\
\hline Data/restraints/parameters & $1400 / 2 / 99$ \\
\hline
\end{tabular}


Tab. 5. Comparison of selected experimental details.

\begin{tabular}{lcc}
\hline & $\mathbf{A}$ & $\mathbf{B}$ \\
\hline Independent reflections & $1400\left[\mathrm{R}_{\mathrm{int}}=0.0413, \mathrm{R}_{\sigma}=0.0235\right]$ & $1400\left[\mathrm{R}_{\mathrm{int}}=0.0341, \mathrm{R}_{\sigma}=0.0213\right]$ \\
Goodness-of-fit on $\mathrm{F}^{2}$ & 1.026 & 1.019 \\
Final R indices $[\mathrm{I} \geq 2 \sigma(\mathrm{I})]$ & $\mathrm{R}_{1}=0.0161, \mathrm{w} \mathrm{R}_{2}=0.0456$ & $\mathrm{R}_{1}=0.0147, \mathrm{wR}_{2}=0.0409$ \\
Final R indices [all data $]$ & $\mathrm{R}_{1}=0.0174, \mathrm{w} \mathrm{R}_{2}=0.0461$ & $\mathrm{R}_{1}=0.0159, \mathrm{w} \mathrm{R}_{2}=0.0413$ \\
Largest diff. peak/hole $\left(\mathrm{e} \cdot \AA^{-3}\right)$ & $0.36 /-0.42$ & $0.33 /-0.25$ \\
\hline
\end{tabular}

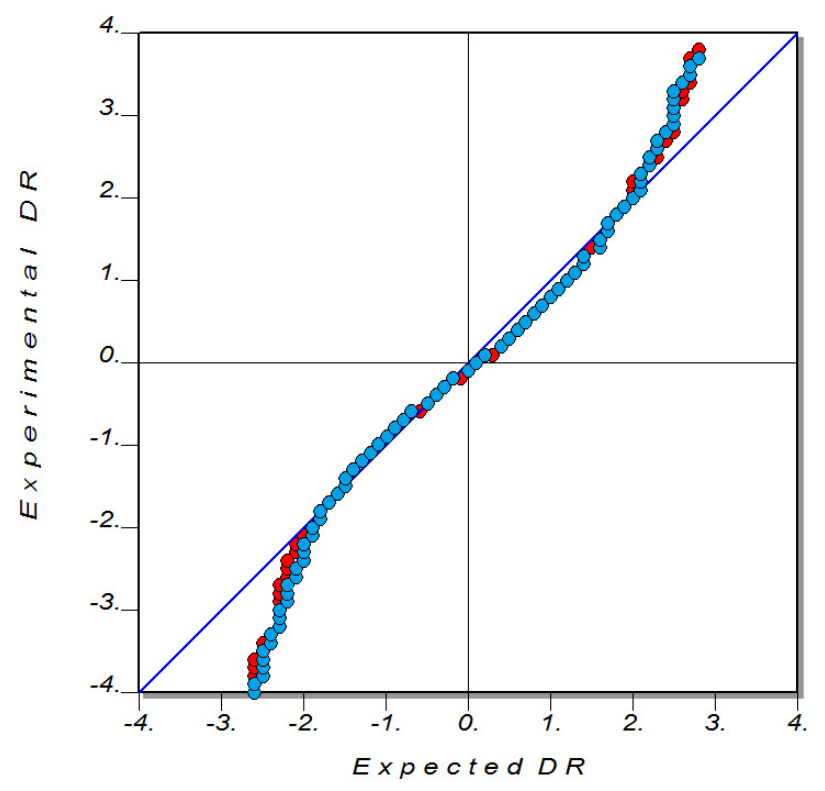

Fig. 2. The normal probability plot with comparison of both absorption correction methods under study (numerical - red, semi-empirical - blue; expected DR “draw” - theoretical quantiles, experimental DR - experimental quantiles)

(Farrugia, 2012).

Error analysis has shown that both refinements exhibit a respectable agreement between model and experimental data. On the normal probability plot (Fig. 2) comparable results for both methods can be seen. The middle part of the plot has linear character and passes the origin with a slope of nearly 45 degrees.

The plot of scale factors for 12 equidistant groups (Fig. 3) are practically identical for both methods. The small differences between scale factors are highlighted by black arrows.

\section{Conclusion}

It was shown that for the routine crystal structure analysis the semi-empirical absorption correction by LANA (with the aid of frame scaling in conjunction with direction-dependent scaling) is preferable (see Table 5). It is not only faster and more user friendly, but also the error analysis shows that the indicators are comparable, or even better. Differences in bond distances and angles are within the standard deviation.

\section{Acknowledgement}

This article was created with the support of the Ministry of Education, Science, Research and Sport of the Slovak Republic within the Research and Development Operational Programme for the project "University Science Park of STU Bratislava”, ITMS 26240220084, co-funded by the European Regional Development Fund and also by support of the Research and Development Agency under the contract No. APVV-15-0079 and Scientific Grant Agency of the Slovak Republic VEGA (Project No. 1/0871/16).

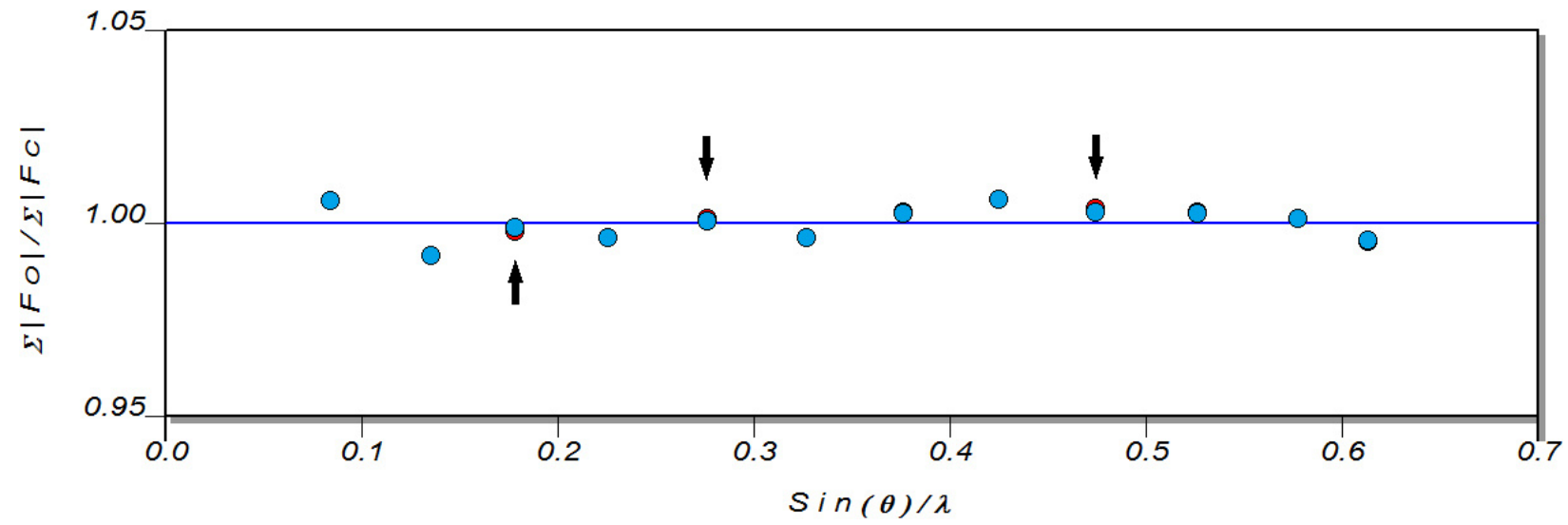

Fig. 3. The plot of scale factors for 12 equidistant groups. Comparison of two different absorption correction methods (numerical - red, semi-empirical - blue) (Farrugia, 2012). 


\section{References}

Bergerhoff G, Berndt M, Brandenburg (1996) J. Res. Natl Inst. Stand. Technol. 101: 221.

Bertolotti F, Forni A, Gervasio G, Marabello D, Diana E (2012) Polyhedron 42: 118.

Blessing RH (1995) Acta Cryst. A51: 33

Dolomanov OV, Bourhis LJ, Gildea RJ, Howard JAK, Puschmann H (2009) J. Appl. Cryst. 42: 339.

Farrugia LJ (2012) J. Appl. Cryst. 45: 849.

Fronczek FR (2003) Private CSD Communication.

Golzar Hossain GM (2007) Private CSD

Communication.

Mahmoudkhani AH, Langer V (1998) Private CSD

Communication.

Nieger M (2001) Private CSD Communication.

Palatinus L, Chapuis G (2007) J. Appl. Cryst. 40: 786.
Shamuratov EB, Batsanov AS, Sharipov KhT, Struchkov YuT, Azizov T (1994) Koord. Khim. (Russ.) (Coord. Chem.) 20: 754.

Sheldrick GM (2015) Acta Cryst. C71: 3.

STOE \& Cie GmbH (2016). X-Area 1.76, software package for collecting single-crystal data on STOE area-detector diffractometers, for image processing, scaling reflection intensities and for outlier rejection; Darmstadt, Germany.

STOE \& Cie GmbH (2013). X-View 2.1.7, program for monitoring and centering a sample on a STOE X-ray diffractometer in combination with a magnifying video camera, and for graphics-supported determination of a crystal-shape model; Darmstadt, Germany.

Vaughan GBM, Schmidt S, Poulsen HF (2004) Z. Kristallogr. 219: 813. 